\title{
University-SME Collaboration and Open Innovation: Intellectual-Property Management Tools and the Roles of Intermediaries
}

\author{
Isabelle Deschamps, Maria G. Macedo, and Christian Eve-Levesque
}

\author{
"Les hommes construisent trop de murs et pas assez de ponts." \\ (Men build too many walls and not enough bridges.) \\ Joseph Fort Newton, Priest and Author \\ as paraphrased by \\ Dominique Pire, Nobel Laureate (Peace)
}

\begin{abstract}
In 2009, the Conseil de la science et de la technologie du Québec (CST) made 13 recommendations to the Government of Quebec in order to shift innovative actors towards open-innovation practices adapted to the province's context: diversified economic sectors, a majority of small and medium-sized enterprises (SMEs), public universities, etc. Among these recommendations are: i) to set up flexible mechanisms to promote research collaboration between public-private sectors such as universities and SMEs, and ii) to optimize intermediation bodies' contribution to establish open-innovation practices. Furthermore, the lack of adequate understanding and tools for the management of intellectual property (IP) was identified as a major inhibitor of open-innovation practices, to which actors should pay specific attention. In this article, we present results and recommendations from a field study focused on two groups of actors: i) companies involved in collaborative innovation and ii) intermediary agents enabling innovation and technology transfer. Our first goal was to shed some light on factors that facilitate open innovation through improved universityenterprise collaborations and, more importantly, that attempt to overcome the irritants related to IP management. Our second goal was to analyze the roles of diverse intermediaries in the fostering of successful collaborations between universities and SMEs.
\end{abstract}

Our study yielded three findings: i) SMEs do not care about understanding and improving their capabilities about IP and are not equipped with adequate tools and best practices for managing IP and for managing the overall collaborative mechanisms in general; ii) this gap in preparation for open innovation is persistent, since even the intermediaries, whose role is to guide SMEs in university-enterprise collaborations, suffer themselves from the lack of appropriate IP transfer and sharing tools, and do not perceive the need to offer better support in this regard; and iii) overall, current IP-transfer and collaboration-management tools are not sophisticated enough to provide appropriate support for the implementation of open innovation, by which we mean more open and collaborative innovation in the context of university-enterprise collaborations. 


\section{University-SME Collaboration: IP Management Tools and the Roles of Intermediaries} Isabelle Deschamps, Maria G. Macedo, and Christian Eve-Levesque

\section{Introduction}

In the current industrialized world, governments and their diverse agencies emphasize the need to increase the propensity and success of open and collaborative innovation (OECD, 2008; tinyurl.com/b3b9kkt; Chesbrough, 2006; tinyurl.com/aqkav9t). The Government of Quebec is no exception, and this emphasis is shared across the rest of Canada as well. In its last Advisory Report, the Conseil de la science et de la technologie du Québec (CST, 2011; tinyurl.com/b9prarq) reinforced the findings from both the Organisation for Economic Cooperation and Development (OECD; oecd.org) and the Government of Canada regarding the poor rates of innovation and success shown by Canadian private enterprises.

One of the important dimensions of successful innovation - beyond sufficient direct investment in academic or private $R \& D$ - is the extent of collaboration between universities and enterprises through technology transfer (e.g., R\&D partnerships, industrial chairs, licenses, and spin-offs). However, Canada performs poorly in this regard (Government of Canada, 2011; tinyurl.com/bes59r9).

Moreover, experts and governments attribute the poor university-enterprise collaborations performance to: i) a large proportion of SMEs in most developed economies, knowing that small businesses show much lower innovation-absorption capabilities than their larger counterparts and ii) a lack of effective technology transfer and flexible IP-management rules from universities. Indeed, the organizational structures and institutional rules of universities are aligned with a long-term vision of knowledge development and diffusion, which tends to inhibit IP transfer and sharing, and is out of phase with SMEs' short-term need for commercialization of innovations (OECD, 2008; tinyurl.com/b3b9kkt).

Those observations renewed our interest in a field study on best practices and factors that facilitate (or inhibit) a more open and collaborative approach to university-enterprise collaborations, and that foster technology transfer, facilitate IP management, and accelerate commercialization of outputs from universityenterprise collaborations, and specifically from university and SME collaborations (CST, 2009; tinyurl.com/ c42jjhu). Based on this fundamental premise, our central research question was: How can intermediaries increase the propensity and the openness of university-enterprise collaborations, and more precisely university and SME collaboration? Subsidiary questions were:

1. How do the SMEs involved in university-enterprise collaborations approach the relationship and the management of IP issues?

2. What is the role of diverse intermediaries in the management of university-enterprise collaborations relationships?

3. What type of tools and management practices are (and should be) used by these intermediaries to better support SMEs in university-enterprise collaborations?

\section{Our Research Mandate}

This article contains data and insights from a Report based on the authors' field research (Deschamps and Macedo, 2011; tinyurl.com/cz6nvmn). The research took the form of: i) four case studies that retrospectively explain, from the point of view of the companies, recent success stories of university-enterprise collaborations in Quebec, and ii) a survey, combining questionnaires and face-to-face interviews with intermediaries and experts from diverse governmental agencies and not-forprofit organizations supporting university-enterprise collaborations. Our mandate was to guide the CST in its recommendations to bodies of the Government of Quebec regarding the methods and tools to be used by intermediaries in managing IP transfer and implementing open-innovation principles during university-enterprise collaborations.

\section{Part I: Case Studies of Innovative SMEs Active in University-Enterprise Collaborations}

\section{Finding 1: Even very innovative SMEs are barely active in terms of searching for IP tools and best practices for collaborative innovation management}

The enterprises under study were very innovative SMEs - they could be considered to be in the top 5\%-10\% of the SME population. They collaborated with universities or research centres on a continual basis, and their top managers considered that they simply had no choice but to be successful in university partnerships and IP transfers. 


\section{University-SME Collaboration: IP Management Tools and the Roles of Intermediaries} Isabelle Deschamps, Maria G. Macedo, and Christian Eve-Levesque

Our case studies of companies involved in universityenterprise collaborations corroborated some issues found in our literature review:

1. SMEs are usually not very active in their search for external information and knowledge, and they very seldom seek out collaborations with universities. According to a recent survey from the Board of Trade of Metropolitan Montreal (2010; tinyurl.com/ck9j6v6), only $10 \%$ of SMEs with more than $\$ 5$ million in sales report an academic collaboration, and only $1 \%$ report a technology transfer.

2. SMEs are, in general, not proactive - even reluctant to be involved in IP protection and management in general (CST, 2009; tinyurl.com/c42jjhu).

3. University-enterprise collaborations are profitable to enterprises of all size, even with cultural and sectorrelated differences.

4. IP management is an irritant to innovative SMEs during contract or license negotiations, but it is not truly an obstacle for the development of a long-term partnership with universities.

5. Innovation success requires that those irritants be appropriately managed with an open-minded approach and appropriate tools. Trust, communication, and complementary objectives and activities are the key factors for the development of a long-term universityenterprise collaborations in the companies under study.

During our interviews with successful entrepreneurs, we found that challenges related to IP management during university-enterprise collaborations vary, but in all instances, they remain an important issue to establish a sound context with their innovation partners, and that the diverse intermediaries involved had played a central role on the settlement of their collaborative relationship with the university or research centre.

We have observed during our own case studies the following set of attitudes and behaviours of entrepreneurs vis-àvis university-enterprise collaborations and IP issues:

1. Even in the case of these very innovative and successful enterprises, the management of university-enterprise collaborations is performed on an ad-hoc basis, in a reactive or defensive mode, when the entrepreneurs are forced to because controversies about IP ownership arose.
2. Entrepreneurs were not proactive; they were not actively looking for information on open innovation or for university-enterprise collaboration "best practices" to improve their relationships or to facilitate the technology transfer.

3. This passive, "no-need-to-improve" attitude persists, even when they realize that it impedes the IP sharing and restrains the information-exchange process during university-enterprise collaborations, which they considered as the basis of their competitive edge.

4. In the long run, they did not look for a fulfilment of this gap in their competencies, mainly because of lack of time, but also because they simply did not know where to find advice in this domain.

In conclusion of our case studies, we inferred that most SME leaders do not have an adequate knowledge of IP management, do not perceive an urgency to improve their capabilities, and do not seek IP training or external advice (i.e., "they don't know what they don't know"). This inference motivated us to better understand how intermediaries could improve the situation and help to break the vicious circle.

\section{Part II: Study of Intermediaries' Roles in University-Enterprise Collaborations}

The collection of primary and secondary data was performed in steps, through three different methods. Firstly, we performed an Internet search with the aim of identifying intermediaries involved in the Quebec Ecosystem of Academic Research and Technology Transfer. We identified nearly 500 entities and categorized them into profiles, according to their direct or indirect intermediary roles during university-enterprise collaborations:

1. Intermediaries and liaison agents within universities (technology-transfer offices)

2. Societies for university technology commercialization

3. Industrial associations

4. Pre-competitive $R \& D$ consortia

5. Collegial technology-transfer centres

6. Government agencies (advisors affiliated with industrial $R \& D$ support programs) 


\section{University-SME Collaboration: IP Management Tools and the Roles of Intermediaries} Isabelle Deschamps, Maria G. Macedo, and Christian Eve-Levesque

\section{Private consultants (specialists)}

8. Research centres and universities

Secondly, a survey was conducted with 26 intermediaries chosen among these entities, having different profiles, acting in diverse roles and industrial sectors. Responses from 15 organizations were analyzed, and representatives from each of the above profiles were in the final sample. Our questionnaire had two parts: i) closed-questions with multiple choices to evaluate the degree of use of diverse IP-management tools and the level of appropriate training to use these tools, and ii) open-ended questions asking intermediaries to identify services offered, links with SMEs, self-reported competencies, contacts, and internal resources.

Thirdly, in order to validate the responses to our survey, we collected contextual and qualitative information during exchanges with major players active in the innovation ecosystem in Quebec. This additional data helped us to establish IP-management best practices; to get a better understanding of gaps, missed links, and related issues; and to interpret our results with a broader perspective.

\section{Listing and classification of IP-management tools and collaboration-management tools in university- enterprise collaborations}

To build our questionnaire, based on our literature review, we established a classification of diverse IP-management and collaboration-management tools useful in university-enterprise collaborations, within a general context of open innovation. Our 17 categories (Box 1) include specific tools and general guides, covering diverse phases of the innovation process and of collaboration relationships, including management practices, tools, and databases useful at both the operational level (e.g., project management, technical issues) and the strategic level (e.g., collaboration objectives, IP sharing, legal issues).

Finding 2: Intermediaries show a very low usage of IP tools and collaboration-management practices during university-enterprise collaborations

Based on our survey, we found that intermediaries show very low-to-medium usage rates of IP-management tools and guides of open-innovation best practices for university-enterprise collaborations. Moreover, most intermediaries apply these tools for their own use only

Box 1. Categories of IP tools, guides, or sources of information useful for IP transfer and open-innovation approaches during a university-enterprise collaboration project

1. Tools to identify nature of the IP

2. Tools to decide or analyze diverse type of IP protection

3. Tools to search for patents

4. Tools to search for technologies

5. Directory of patents/technologies/expertise available for technology transfer

6. Tools for the management of IP rights/IP ownership

7. Tools for the monitoring of industrial sectors and norms

8. Guides for the commercialization of IP

9. Standard models of university-enterprise contracts and licenses

10. Guides for collaboration with universities

11. Guides for collaboration with enterprises

12. Guides on principles and good practices of open innovation

13. Guides on the financing of collaborations and technology transfers

14. Best practices of transfer agents

15. Publications/journals/specialized review/books

16. Internet blogs on litigation/case studies

17. Guides on where to find important information/references 


\section{University-SME Collaboration: IP Management Tools and the Roles of Intermediaries} Isabelle Deschamps, Maria G. Macedo, and Christian Eve-Levesque

and do not diffuse those tools to SMEs, except for one governmental agency that directly supports SMEs and a few intermediaries, such as R\&D consortia.

Degrees of usage of tools and management practices used by intermediaries are as follows:

1. Traditional monitoring tools that help intermediaries stay informed about technology and industry trends in general but not specific for IP management $(50 \%$ usage)

\section{Patent-management tools (30\% usage)}

3. Sophisticated tools associated with open-innovation practices, commercialization modes, and IP litigation ( $15 \%$ usage)

Finding 3: Intermediaries perceive no need for improving IP tools and collaboration practices

A very low proportion of intermediaries reported a need for change in their role during university-enterprise collaborations, and they did not anticipate any improvement. Only $12 \%$ of all interviewees reported plans to acquire or to develop new tools in a near future, and less than $10 \%$ reported plans to provide their personnel with training on those tools.

General Insight 1: Intermediaries active in universityenterprise collaborations perceive themselves as generalists and they are reluctant to be involved when facing IP and collaboration issues

This lack of motivation by intermediaries to offer better university-enterprise collaboration support and IP tools to SMEs seems to be related to two main factors. First, there is an external perception of a scarce demand of support from SMEs, this perception being consistent with our precedent findings of a passive attitude of SMEs towards IP issues. Second, there is an internal perception by intermediaries that providing tools and support to SMEs is not part of their mandate. Some of our respondents even emphasized that their mandate was the opposite: to represent and defend the interests of the university during the negotiation of IP agreements in university-enterprise collaborations.

In search for an explanation for the low usage of IP tools and collaboration-management best practices inspired by open-innovation principles, we infer from our analysis that most intermediaries consider themselves as generalists. IP tools and collaboration manage- ment are neither perceived as one of their specialities, nor as parts of their mandate. This negative perception might explain their very low propensity to search for, learn, use, and master more effective IP-management and collaboration-management tools.

General Insight 2: Specialists, mainly private consultants, play positive but limited roles for SMEs

Since most intermediaries, considering themselves as generalists, and prefer to avoid involvement with SMEs, IP-management issues are most often left in the hands of specialists, such as technical analysts, legal negotiators, and professional collaboration and open-innovation trainers (e.g., patent agents, lawyers, IP consultants and brokers, management firms).

Our study highlights that the actual main sources of specific advice for SMEs in terms of IP management and university-enterprise collaborations are private consultants (technology brokers, patent agents, etc.); they correspond to the seventh profile in our earlier classification of intermediaries. Some consultants bring state-of-the-art and available knowledge on business or legal dimensions, whereas others bring more technical expertise or specific information about IP transactions and litigations.

This presence of specialists seems to be positive, from the point of view of our respondents; however, the scope of their intervention is restrictive in terms of general impact on university-enterprise collaborations at large:

1. The number of private consultants is limited and they normally prioritize large enterprises; they have less time available for SMEs.

2. They are practically absent outside metropolitan areas.

3. Their services are expensive for SMEs, which have normally a limited budget.

4. They are often too specialized and SMEs do not know how to communicate with them.

5. They tend to focus on specific issues or problems, not considering the general context and the building of the university-enterprise relationships.

6 . They work on a case-by-case basis, and are not preoccupied by the systemic nature of university-enterprise collaborations. 


\section{University-SME Collaboration: IP Management Tools and the Roles of Intermediaries}

Isabelle Deschamps, Maria G. Macedo, and Christian Eve-Levesque

\begin{abstract}
General Insight 3: Complementary profiles of intermediaries (generalists and specialists) are involved at diverse phases of the innovation process
\end{abstract}

As a general insight, we infer that it is important to better analyze and comprehend the relative and complimentary roles of intermediaries in university-enterprise collaborations, in order to fulfill the multiple needs of SMEs in terms of management tools and guides. We conclude that it is important to carefully separate the respective roles of the generalists who play a general management advice role, versus IP specialists, the latter group coming into play later on in the university-enterprise collaboration process. Based on our case studies with successful entrepreneurs (Part I) and our survey of intermediaries (Part II), it is clear that a specific attention should be given to enrich the role of the generalist intermediaries, who are active upstream in the innovation process, and therefore act early on in the establishment of a collaborative climate.

The following consensus emerged after our survey and our consultation with experts active in the innovation ecosystem: front-line generalists do not need to fully master the sophisticated tools in order to perform the complete analysis in terms of IP management or patentability on their own. However, they do need to understand enough about the specific university-enterprise collaboration context in each project, upstream of the innovation process, in order to identify at an early stage the potential IP issues at stake. Furthermore, they should aim to establish a common agenda and language between the two parties.

This role of front-line intermediaries is a key one, because it is presumed that it will fuel positive and more open-minded discussions and exchanges between SMEs and universities. Moreover, a positive context sets up a framework for discussion and collaboration between the generalists and specialists, the latter group being involved during the multiple transactions, as an innovation project unfolds. For example, in Quebec, $R \& D$ consortia are typically involved first, college technology-transfer centres are second, and private consultants are third.

General Insight 4: A lack of knowledge and a poor mastering of the best practices by intermediaries limit the propensity of SMEs to engage in university-enterprise collaborations

We infer from our multiple conversations with experts and actors in Quebec's innovation ecosystem that very little systematic and collective effort is made by all players to increase the number of university-enterprise collaborations involving SMEs and to improve their levels of success. Intermediaries do not significantly use guides for collaboration in order to establish a systematic and more standardized method of building numerous, sound, and long lasting university-enterprise collaborations. When SMEs look for a university collaborator, intermediaries emphasize informal and personal networks of contacts. This decreases the probability of finding the right partner, and reduces the number of university-enterprise collaborations initiated. Overall, this impedes the establishment of the most-needed SMEs' orientation towards open and collaborative innovation. With very little support, and because they are naturally passive about collaboration, SMEs are more likely to show a very low sense of urgency for expanding their collaboration networks, for learning new open-innovation management practices for university-enterprise collaborations, and for absorbing innovations from external sources.

General Insight 5: A lack of front-line intermediaries' expertise and support with innovative SMEs impedes collaboration, fuels distrust, and leads to lost opportunities

The relatively static and "closed" state of mind about collaborative innovation, shown both by SMEs and intermediaries, including liaison agents within universities, is quite troubling. The globalizing industrialized world is continuously accelerating the rate of IP exchange all around the world. In such a context in favour of open innovation and IP transactions, growing opportunities for collaborations arise, both for SMEs and universities. From a local socio-economic development point of view, the actual low level of university-enterprise collaborations represents an enormous loss of potential.

Generalists who are dispersed throughout the territory and who act as front-line intermediaries during university-enterprise collaborations are naturally involved in early discussions related to IP. At that stage, they should master some basic IP-management concepts, perhaps less than specialists such as patent agents, but at least more than average SMEs. The reported lack of knowledge about IP-related issues among SMEs inhibits university-enterprise collaborations - it is a source of distrust (Board of Trade of Metropolitan Montreal, 2010; tinyurl.com/ae828tx). If most intermediaries know almost nothing about IP management, as reported in our survey, it becomes highly probable that any type of IP subject matter will easily become a dispute, due to lack of appropriate knowledge to solve the raised questions. 


\section{University-SME Collaboration: IP Management Tools and the Roles of Intermediaries}

Isabelle Deschamps, Maria G. Macedo, and Christian Eve-Levesque

Front-line intermediaries, even if they are generalists, must be knowledgeable and reassuring with SMEs, so they need to grasp a minimal level of knowledge and to build self-confidence in their mediator's role in order to increase mutual trust and credibility.

\section{Conclusion}

Conclusion 1: A systemic approach for collaboration is needed, beyond fixing symptoms and solving IP-related problems between SMEs and universities.

Our analysis of practices in the Province of Quebec reveals multiple challenges related to IP management in university-enterprise collaborations and proposes some avenues to reinforce the roles of intermediaries and to diffuse some "best practices" about the management of IP. Each of those challenges must be perceived as part of a whole, in which IP is only one aspect.

Our analysis of intermediaries' profiles and attitudes clearly depicts a lack of pro-activity and reveals gaps in knowledge of appropriate tools. This attitude represents a strong barrier to the implementation of open-innovation principles in university-enterprise collaborations. This situation might also explain the very low proportion of SMEs that are likely to engage in university collaboration: the entrepreneurs interviewed during our case studies reported that they feel that most of intermediaries are either too passive to support them or even working against them. Of course, this does not imply that all universities and intermediaries are behaving in the same way; on the contrary, interviews confirmed the existence of wide spectrums of support from intermediaries, collaboration attitudes, and IP-management rules in universities.

\section{Conclusion 2: More open and collaborative innovation is possible in university-SME contexts if intermediaries play a more proactive and opportunity-driven role.}

Our study leads us to strongly believe that the simultaneous implementation of IP-management tools and a better understanding of general collaboration-management issues could create a virtuous circle of improvement, towards more successful university-enterprise collaborations and better open-innovation management practices. A rapprochement of SMEs with universities, through upstream intermediaries who would be better prepared to foster a positive climate for match-making, could stimulate a willingness to collaborate from both parties. A better and reciprocal matching of opportunities would set the stage for more productive discussions, and would encourage intermediaries to invest in more sophisticated networks, tools, and practices to manage university-enterprise collaborations. In particular, better tools are needed to analyze the stakes and the respective IP positions upstream in order to facilitate dialogues and accelerate negotiations. Overall, win-win agreements would be reached, universities would appear more open to the community of SMEs, and the proportion of SMEs willing to be involved in university-enterprise collaborations would increase.

\section{Conclusion 3: Generalists and specialists play two inextricably interrelated roles.}

Based on the results of our survey, we can observe two categories of intermediaries: i) front-line generalists and ii) specialists. The first group is diverse in nature, and most of them work for governmental agencies or not-for-profit organizations; the latter group is mainly composed of private consultants. It would be very important to carefully distinguish the respective roles of front-line generalists from those of specialists given that there is currently a significant gap in the level of knowledge in terms of IP-management tools. During first phases of an innovation project, SMEs generally turn to generalist intermediaries for support, but these intermediaries know almost nothing about complex IP issues. There is a need to raise the level of knowledge of those front-line intermediaries who could be subsequently able to refer SMEs to appropriate specialists and to work in cooperation with them - as a complementary team. In order to allow specialized private consultants to offer useful services to SMEs, which are specific and customized to their project, all front-line intermediaries must master at least a basic understanding of the content, vocabulary, and usefulness of specialized services offered. This knowledge is especially crucial for referring the SMEs in a very timely manner to the appropriate specialist in IP protection and transfer, because a lack of upstream preparation in this matter significantly slows down the collaboration process between SMEs and universities in downstream activities, and it could lead to disputes that will jeopardize the commercialization phases. 


\section{University-SME Collaboration: IP Management Tools and the Roles of Intermediaries} Isabelle Deschamps, Maria G. Macedo, and Christian Eve-Levesque

\section{Recommendations}

Based on our general observations and overall analysis of their roles, it appears that all intermediaries, and especially the front-line generalists, should not only master the basics of IP-management tools, but they should also become liaison agents - even coaches or mediators - between universities and SMEs. We offer the following recommendations to put in place such measures:

1. Financing agencies that support intermediaries must consider the expansion of intermediaries' capabilities and roles, and eventually renew their missions as well as their financing and business models. Intermediaries, and especially the front-line generalists, could more directly and effectively support SMEs in their search for more open and collaborative university-enterprise collaborations.

2. Government agencies would benefit to envision a reorganization of roles and responsibilities of intermediaries, including generalists and specialists, which should evolve towards more pro-activity in terms of usage and diffusion of best practices in IP and collaboration management. However, those changes cannot be implemented on a case-by-case basis. Intermediaries are dependent on one another. Governments must aim to create an integrated chain of intermediaries with complementary profiles and common practices, to better support SMEs at all phases of the innovation process.

3. This integration requires closer interactions between diverse government levels (i.e., federal, provincial, local or regional, sector-specific), universities, intermediaries, and SMEs in order to harmonize rules of IP sharing and trading as well as to better integrate stakeholders so that they work together and create synergy throughout the chain of technological innovation.

Implementing these recommendations requires a global and systemic approach; otherwise, it is possible that isolated changes, on specific IP rules or mechanisms aimed to improve some collaboration activities at early innovation phases, could have a counter-productive effect at subsequent steps of the open and collaborative innovation process.
The objective of those new or improved methods discussed above is not primarily quantitative, but qualitative. Beyond the desire to increase the absolute amount of university-enterprise collaborations and to speed up negotiations, the objective is to improve - in a sustainable manner - the capacity of SMEs to manage innovation in an open and collaborative way with university partners. We collectively need SMEs that are able to absorb and create IP in order to generate the maximum commercial outputs from all sources of IP, and especially form local universities. To accomplish this, our innovation ecosystem needs to rely on well-prepared intermediaries with expanded roles.

As a final note, since data was collected in 2010, we have observed that some intermediaries have improved on some of their services, support mechanisms, and tools. It would be interesting to repeat our survey and measure progress along the suggested lines in our conclusions and recommendations.

\section{About the Authors}

Isabelle Deschamps is Professor and founder of graduate programs in Innovation Management at École de technologie supérieure in Montreal, Canada. A professional engineer, she has been active for 30 years as a consultant, trainer, investor, and researcher in the fields of collaborative technological innovation. She has been advisor and manager in governmental agencies, incubators, transfer centres, and clusters in industries such as aluminum, environment, information technologies, nanotechnologies, and life science. Directly active for more than 15 years in the Quebec innovation ecosystem, she coached and financed startups and university spinoffs as Vice-President of Inno-centre, a technological incubator and as Partner of Capimont Technologies, a private venture capital group. Since 2007, she has been involved in fast-growing high-tech SMEs for the management of innovation and universityenterprise collaborations. She holds an MBA from HEC-Montréal and a DBA in Innovation Management from Harvard University.

\section{Continued on next page...}




\section{University-SME Collaboration: IP Management Tools and the Roles of Intermediaries} Isabelle Deschamps, Maria G. Macedo, and Christian Eve-Levesque

\section{About the Authors (continued)}

Maria Macedo is a Technology Transfer Officer at Service des partenariats et soutien à l'innovation (SePSI-UQAM), in Montreal, Canada, where she manages partnership research projects and university intellectual property. In this position, she creates a link between top university research and the unmet needs of industry, thereby providing the university's members with a real opportunity to gain any commercial benefits arising from their research. Maria has over 10 years of experience in innovation management and strategic planning. In recent years, she has conducted industry studies that identify the strengths, weaknesses, opportunities, and threats to the industry's development and innovation. Maria completed her undergraduate studies at Universidad Nacional Autonoma de México (UNAM) in Food Chemistry and she holds an MSc and a PhD in Food Sciences from Laval University in Québec City as well as a MBA from HEC Montréal. She is finalizing an MSc in management of innovation at École de technologie supérieure in Montreal.

Christian Eve-Levesque is a young professional who cumulates experience in product development, technology transfer, and production engineering in medical and robotic sectors. He holds a Bachelor of Mechanical Engineering and a Masters Degree in Innovation Management from École de technologie supérieure in Montreal, Canada. He is active in university-enterprise collaboration projects as well as in enterprise-enterprise collaboration projects. Christian also teaches business strategy for graduate programs in Innovation Management at École de technologie supérieure.

Citation: Isabelle Deschamps, I., M.G. Macedo, and C. Eve-Levesque. 2013. University-SME Collaboration and Open Innovation: Intellectual-Property Management

Tools and the Roles of Intermediaries . Technology

Innovation Management Review. March 2013: 33-41.

Keywords: open innovation, university-SME

collaboration, university-enterprise collaboration,

intellectual property, IP management, technology

transfer, intermediaries 